\title{
Estudo do Envelhecimento de Poliuretanos Aplicados na Indústria de Petróleo
}

\author{
Fabio G. Aquino, Terry Sheldrake, Judimar Clevelario, Fabio Pires \\ Wellstream International \\ Fernanda M. B. Coutinho ${ }^{\dagger}$ \\ Instituto de Química, UERJ
}

\begin{abstract}
Resumo: O Brasil é mundialmente renomado como um líder na extração de petróleo, em águas profundas e ultra profundas. Dentro da cadeia produtiva, grande parte do petróleo e do gás produzido é escoada através de dutos flexíveis que conectam os poços de produção às plataformas. Quando estão apoiadas no fundo do mar, em condição de serviço estático, os dutos flexíveis são denominados flowlines e quando se elevam do fundo do mar até a plataforma, em condição de serviço dinâmico, são denominados risers. Os tubos projetados para aplicações dinâmicas são dotados de bends stiffeners, componentes com formato cônico e, em geral, de base uretânica que têm a função de fornecer uma transição entre a estrutura dos tubos, de rigidez suave, e a plataforma, extremamente rígida. Este trabalho abordou a variação das propriedades mecânicas de poliuretanos como resultado da ação da hidrólise, por envelhecimento acelerado. Os corpos de prova foram expostos a 50, 60 e $70{ }^{\circ} \mathrm{C}$. A variação de massa também foi determinada considerando que esses materiais são projetados para uma vida útil superior a vinte anos. Os poliuretanos apresentaram degradação significativa a $70{ }^{\circ} \mathrm{C}$, o que pode reduzir seu tempo de aplicação no campo por falha mecânica quando submetido a elevadas temperaturas.
\end{abstract}

Palavras-chave: Degradação, poliuretanos, propriedades mecânicas, variação de propriedades.

\section{Study of Ageing of Polyurethanes Applied to the Offshore Industry}

Abstract: Brazil is worldwide renowned as a leader in oil and gas extraction in deep and ultra deep water. In the production chain, a great part of the oil and gas produced is conveyed through flexible pipelines that connect the production wells to the platforms. When the pipes are laid on the seabed in a static service condition, the flexible pipes are called flowlines and when raised from the seabed to the platform in a dynamic service condition, they are called risers. The pipes designed for dynamic applications are equipped with bend stiffeners, components with conical form and in general based on polyurethanes, which have the function of providing a transition between the structure of the pipes, of smooth stiffness, and the platform, extremely rigid. This work discussed the changes on the mechanical properties of polyurethanes due to hydrolysis under accelerated ageing. The specimens were exposed at 50,60 and $70{ }^{\circ} \mathrm{C}$. The mass variation was also evaluated considering that these materials are designed for a service life exceeding twenty years. The polyurethanes presented significant degradation at $70{ }^{\circ} \mathrm{C}$, which can reduce its application time in the field owing to mechanical failures when subjected to high temperatures.

Keywords: Degradation, polyurethanes, mechanical properties, properties variation.

\section{Introdução}

A evolução e as necessidades da vida moderna levam o homem a buscar novas fontes para a pesquisa e, até pouco tempo atrás, era importante descobrir materiais cada vez mais duráveis para a utilização diária. Dentre esses estavam os polímeros, com grande variedade de aplicações, devido às suas propriedades, versatilidade e preço ${ }^{[1]}$. Dentre as classes e tipos de polímeros estudados, destacam-se os poliuretanos, desenvolvidos por Otto Bayer, em 1937, na Alemanha ${ }^{[2]}$. Inicialmente, essa classe de polímeros destinou-se a concorrer com a poliamida (Nylon) descoberta por Carothers da Du Pont, EUA ${ }^{[3]}$. No final da década de 1930, na Alemanha, iniciou-se a comercialização dos poliuretanos para a fabricação de adesivos, tintas, cerdas, fibras e espumas rígidas ${ }^{[4]}$.

No decorrer da década de 1940, apareceram na Inglaterra e na Alemanha os elastômeros de poliuretanos. Em meados da década de 1950, ocorreu um avanço comercial com o desenvolvimento da espuma flexível. Na década de 1960, o uso do clorofluorocarbonetos, CFC, como agente de expansão na produção de espuma rígida resultou em uma extensa aplicação desse material como isolantes térmicos.

\footnotetext{
$\dagger$ in memoriam
} 
A espuma semi-rígida, de base uretânica, revestida com material termoplástico, destinada à indústria automobilística, foi desenvolvida na década de 1970 e na década de 1980, surgiu a moldagem por injeção reativa (RIM), com grande crescimento comercial.

Na década de 1990, agravou-se o problema com o meio ambiente, que levou ao desenvolvimento da pesquisa voltada para a substituição do CFC, considerado danoso à camada de ozônio. $\mathrm{Na}$ atualidade, o maior destaque nessa área é a moldagem por injeção reativa e a pesquisa relacionando a estrutura molecular às propriedades dos poliuretanos. A tecnologia dos poliuretanos tornou-se assim, uma fantástica história de sucesso e um negócio de muitos bilhões de dólares $^{[5]}$.

Os poliuretanos são uma classe notável de polímeros que exibem uma larga faixa de propriedades mecânicas, tornando-os muito versáteis. Dependendo do catalisador e dos monômeros utilizados, pode ser gerada uma grande variedade de materiais, com estrutura maciça ou celular.

Poliuretanos podem ser produzidos em duas formas: termoplásticos e termorrígidos. Dentre as propriedades específicas dos poliuretanos, a boa resistência à abrasão e o fácil processamento são destaques para esse material ${ }^{[2]}$.

Os poliuretanos estão correntemente sendo aplicados em serviços marinhos. Na indústria de petróleo, essas aplicações incluem camadas para proteção de linhas em águas profundas. $\mathrm{Na}$ indústria de pesca outros tipos de poliuretanos vêm sendo propostos. Assim como aplicações em oceanografia, como camadas de proteção para veículos subaquáticos ${ }^{[6]}$. Esses materiais se destacam pelo alto tempo de vida útil em campo, continuamente e intermitentemente. $\mathrm{O}$ material selecionado deve manter suas propriedades mecânicas durante o período da aplicação, em condições ambientais.

Há estudos sobre a durabilidade dos poliuretanos quando expostos às condições de aplicações marinhas. Por exemplo, os procedimentos apresentados em um simpósio da ASTM sobre materiais para aplicações em águas profundas, realizado em 1969. Nesse evento foram incluídos alguns dados sobre o comportamento de polímeros comuns ${ }^{[7]}$. Vários mecanismos que contribuem para a durabilidade de polímeros adesivos em presença de água foram descritos ${ }^{[8]}$. Esses incluem plastificação, inchamento, hidrólise, etc. Compósitos com fibras reforçadoras têm sido amplamente usados em estruturas marinhas. Uma revisão recente sobre a durabilidade de lâminas marinhas indicam uma extensão para materiais termorrígidos ${ }^{[9]}$.

Dados obtidos com amostras retiradas de estruturas marinhas, imersas no mar por 20 anos, foram correlacionados com resultados de ensaios acelerados em laboratório ${ }^{[10]}$. Parâmetros de perda vêm sendo utilizados para avaliar a influência da água do mar no que se refere ao envelhecimento de polímeros em seus compósitos ${ }^{[1]}$. Curvas mestres com base em tempo-temperatura estão sendo utilizadas para avaliar a variação das propriedades de compósitos em água do mar ${ }^{[12]}$.
Outros estudos incluem o envelhecimento e tempo de vida de elastômeros, assim como seus efeitos ${ }^{[13]}$. Muitos desses estudos se referem ao envelhecimento ao ar, onde a fotodegradação é o principal foco. Contudo, poucos autores têm publicado resultados sobre o envelhecimento em água do mar. O modelo de envelhecimento para borracha natural, neopreno e poliuretano, utilizando água do mar artificial por um período de 2 anos foi utilizado ${ }^{[14]}$. As propriedades de rigidez do poliuretano foram estáveis até o final dos testes. Murata et al. ${ }^{[15]}$ discutiram a resistência à hidrólise de poliuretanos. Os autores mostraram a importância dos grupos hidroxíla na estabilidade da massa molar em água ${ }^{[15]}$. Rutkowaska et al. ${ }^{[16]}$ examinaram a degradação de poliuretano à base de poliéster e poliéter em água do mar por um período de 12 meses. Os autores mostraram a maior resistência do último ${ }^{[16]}$. Uma revisão recente sobre elastômeros usados na indústria de petróleo descreve diferentes modelos de degradação, incluindo a degradação mecânica, química e rompimento de ligações devido ao ataque por ação de raios UV ${ }^{[17]}$.

A técnica mais comum para acelerar o envelhecimento de materiais aplica testes com temperatura elevada. A expressão de Arrhenius é usada para avaliar o material em baixas temperaturas ou tempos longos. Esse procedimento está descrito na norma ISO 11346 de 1997. Entretanto, a validade dessa técnica envolve uma discussão considerável. $\mathrm{O}$ uso do teste utilizando temperaturas elevadas de forma a acelerar o envelhecimento é baseado na suposição da cinética do mecanismo de envelhecimento na faixa de temperatura empregada. Celina et al. ${ }^{[18]}$ examinaram a análise de Arrhenius para uma série de materiais expostos ao envelhecimento térmico ${ }^{[18]}$. Os autores reforçaram a necessidade de se estabelecer uma extrapolação linear válida e mostraram que determinados mecanismos podem resultar em um comportamento anti-Arrhenius. Outro estudo, usando policloropreno envelhecido em laboratório e 24 anos de em serviço no campo, também mostrou evidências de que a curva de extrapolação linear de Arrhenius não é válida ${ }^{[19]}$.

A influência do envelhecimento está relacionada com as propriedades mecânicas do material. Essas propriedades são facilmente monitoradas. Os testes de compressão, tração, dureza, fadiga e resistência de selagem podem ser aplicados ${ }^{[20]}$. Outros métodos podem ser utilizados em paralelo com testes mecânicos, como a espectroscopia na região do infravermelho (FTIR), variação de massa e valores de permeabilidade, assim como cromatografia de exclusão por tamanho (SEC) e ressonância magnética nuclear (NMR).

FTIR é uma técnica bastante empregada para determinação de variações na estrutura química de poliuretanos. Mudanças significativas vem sendo observadas em ligações químicas para acompanhar diferenças de separação de fases. Lee at al. ${ }^{[21-23]}$ monitoraram a separação de fases de um polieter-uretano modelo usando FTIR e DSC, pelo monitoramento das intensidades relativas às bandas de carbonilas livres nas cadeias.

Devido ao fato das linhas flexíveis estarem inseridas em um ambiente aquático marinho, há a possibilidade de 
que materiais de base uretânica venham a sofrer algum dano estrutural devido à presença de água como agente de hidrólise, principalmente associado ao trabalho envolvendo temperatura externa e até mesmo a temperatura interna do fluido por gradiente de temperatura. Assim, a hidrólise, fenômeno de despolimerização, resultante da reação química entre a água no estado líquido ou gasoso (vapor) e as cadeias do polímero, a formação de foto produtos, a alteração da massa molar do poliuretano, entre outros, podem levar esses materiais poliméricos à falha estrutural.

O objetivo deste trabalho foi estudar a variação das propriedades mecânicas de poliuretanos aplicados na indústria de petróleo quando submetidos a condições de hidrólise, por envelhecimento acelerado e absorção de água do mar.

\section{Experimental}

\section{Materiais e métodos}

Poliuretanos à base de poli(tetrametileno glicol) e diisociantao de tolileno (PTMEG/TDI), denominados PU-1 e PU-2 tiveram a sua resistência à tração avaliada de acordo com o método ASTM D638-09. Foram feitas medidas contínuas de resistência desenvolvida pelo material polimérico quando este sofria alongamento sob razão constante de deformação. A procedência dos poliuretanos é de natureza sigilosa.

As propriedades tênseis foram medidas em máquina de ensaios universal - Instron, modelo 5569 com célula de carga de $2 \mathrm{kN}$. Os ensaios foram realizados a $10,20,50$ e $70{ }^{\circ} \mathrm{C}$ para se acompanhar o comportamento dos poliuretanos com a variação de temperatura de aplicação em campo. Foram ensaiados 10 corpos de prova por nível de temperatura, sendo estes obtidos segundo método ASTMD638 - 03 - Tipo 4. As análises foram conduzidas com velocidade de estiramento de $50 \mathrm{~mm} / \mathrm{min}$. Os corpos de prova foram acondicionados na câmara acoplada ao equipamento de ensaio, sendo o teste iniciado após 50 minutos de estabilização na temperatura de realização do ensaio. Os resultados obtidos a $20{ }^{\circ} \mathrm{C}$ foram utilizados como valores de referência para os ensaios de envelhecimento acelerado.

\section{Propriedades de envelhecimento}

Foi realizado teste de estabilidade hidrolítica em amostras de poliuretanos de acordo com a norma ASTM D3137. Os corpos de prova foram imersos em água destilada em diferentes temperaturas: 50,60 e $70{ }^{\circ} \mathrm{C}$ e acondicionados em estufa da marca Fanem, modelo Ret 270. Para cada temperatura, foi avaliada a influência do tempo de exposição: 250, 500, 1000, 2000, 3000, 4000 e 5000 horas. Para cada condição de exposição (tempo-temperatura) foram utilizados durante os ensaios 10 corpos de prova para cada tipo de poliuretano. Os corpos de prova tiveram suas propriedades mecânicas avaliadas em máquina de ensaios universal Instron, modelo 5569. O objetivo do teste foi verificar a variação das propriedades mecânicas dos poliuretanos sob a ação da água (hidrólise) por envelhecimento acelerado.

Os testes de tração foram realizados de acordo com a norma ASTM D638-09, para avaliar o comportamento mecânico dos poliuretanos antes e após cada período de exposição. As propriedades mecânicas dos poliuretanos foram determinadas com a aplicação de uma taxa de deformação de $50 \mathrm{~mm} / \mathrm{min}$. Os corpos de prova foram moldados de acordo com a norma ASTM D638-03 - Tipo 4.

\section{Variação de massa}

Cada corpo de prova teve sua massa verificada antes e após cada período de envelhecimento de modo a se verificar alguma diferença entre as mesmas. As medidas foram realizados a temperatura ambiente.

\section{Resultados e Discussão}

A análise do comportamento mecânico de um material polimérico se baseia no estudo das deformações causadas pela aplicação de forças externas. A aplicabilidade final de um material polimérico é, principalmente, ditada por suas propriedades mecânicas. Essas propriedades, por sua vez, são fortemente dependentes do nível de homogeneidade, compatibilidade e de adesão entre os componentes do sistema $^{[24]}$.

A resistência à tração na ruptura e o alongamento na ruptura podem ser correlacionados quantitativamente com a estrutura do polímero. Materiais com alto teor de ligações cruzadas, como os utilizados nas espumas rígidas, são fortes e duros, porém quebradiços, e os elastômeros e as espumas flexíveis apresentam alongamento na ruptura muito maior. Uma outra maneira de estudar o comportamento mecânico dos poliuretanos está relacionada com o processo de síntese do polímero, uma vez que o aumento de segmentos rígidos na polimerização, ou seja, o aumento do teor de isocianato na formulação do material leva ao aumento do módulo e diminuição do alongamento na ruptura do mesmo ${ }^{[25]}$.

Os testes de resistência à tração dos poliuretanos selecionados para este trabalho foram realizados a 10, 20, 50 e $70{ }^{\circ} \mathrm{C}$, de modo a se verificar o comportamento dos materiais com a variação de temperatura. As Figuras 1 e 2 apresentam os resultados de alongamento médio na ruptura e tensão média na ruptura.

De um modo geral, o poliuretano identificado como PU-1 apresentou tendência de estabilização dos resultados de alongamento até a temperatura de $50{ }^{\circ} \mathrm{C}$, seguida de uma queda de aproximadamente $134 \%$ com a elevação da temperatura de teste para $70{ }^{\circ} \mathrm{C}$. O polímero identificado como PU-2 mostrou uma tendência de elevação dos valores de alongamento com o aumento da temperatura de teste, de um modo em geral, assim como uma maior estabilidade nas propriedades de alongamento quando comparado ao PU-1. Os valores superiores de alongamento na ruptura para o PU-1, quando comparados aos do PU-2, podem estar 


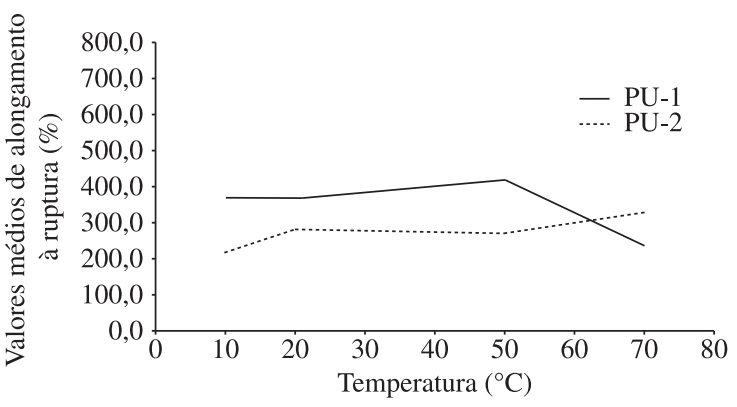

Figura 1. Valores médios de alongamento na ruptura.

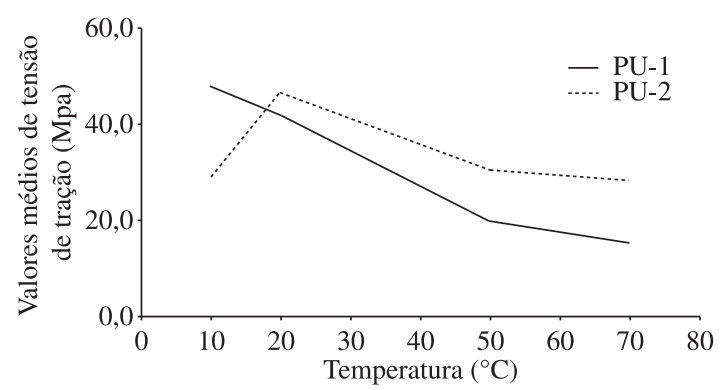

Figura 2. Valores médios de tensão na ruptura.

correlacionados com o teor mais baixo de segmentos rígidos na estrutura desse poliuretano ${ }^{[25]}$, uma vez que a estrutura dos materiais é de natureza desconhecida. Esse comportamento é confirmado pelos resultados de tensão na ruptura (Figura 2) nos ensaios realizados a 20,50 e $70{ }^{\circ} \mathrm{C}$, pois os valores de tensão na ruptura maiores foram encontrados para o PU-2.

O teor de ligações cruzadas também é outro fator que pode explicar o comportamento mecânico dos poliuretanos, uma vez que quanto maior o número de ligações cruzadas na estrutura do polímero, maior será o módulo de tração na ruptura do mesmo.

Com relação aos valores médios de tensão na ruptura, o poliuretano identificado como PU-1 mostrou acentuada tendência para decréscimo com o aumento da temperatura de teste. Com relação ao PU-2, foi verificada a elevação dos resultados a $20^{\circ} \mathrm{C}$, seguida de acentuada redução dos valores de tensão de ruptura com a elevação da temperatura de teste. Comparando os resultados a 10 e $70{ }^{\circ} \mathrm{C}$ para o PU-2, foi evidenciada uma similaridade entre os mesmos, verificandose uma maior estabilidade para o PU-2 com a variação da temperatura. Com relação ao PU-1, houve redução nas propriedades tênseis com o aumento de temperatura, mostrando uma maior limitação desse material em aplicações sob temperatura elevada.

\section{Estabilidade hidrolítica}

Os valores de resistência à tração realizado a $20{ }^{\circ} \mathrm{C}$ foram usados como referência para o início dos testes de envelhecimento.

As Figuras 3, 4 e 5 apresentam os valores médios de alongamento na ruptura para os corpos de prova envelhecidos até 5.000 horas, submetidos a temperatura de 50,60 e $70{ }^{\circ} \mathrm{C}$,

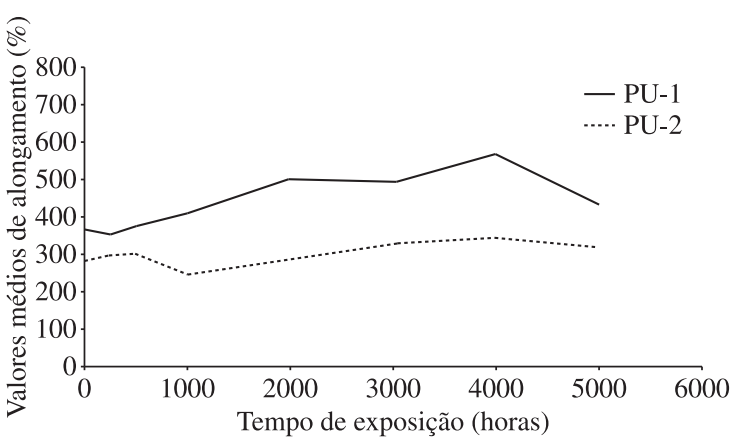

Figura 3. Valores médios de alongamento na ruptura a $50{ }^{\circ} \mathrm{C}$ - Hidrólise.

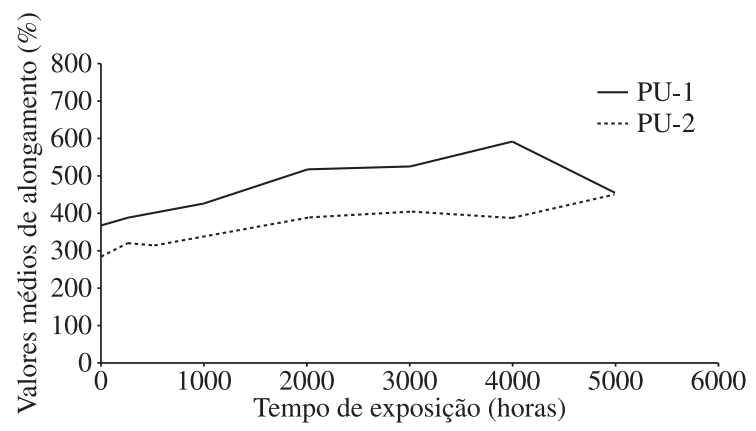

Figura 4. Valores médios de alongamento na ruptura a $60{ }^{\circ} \mathrm{C}$ - Hidrólise.

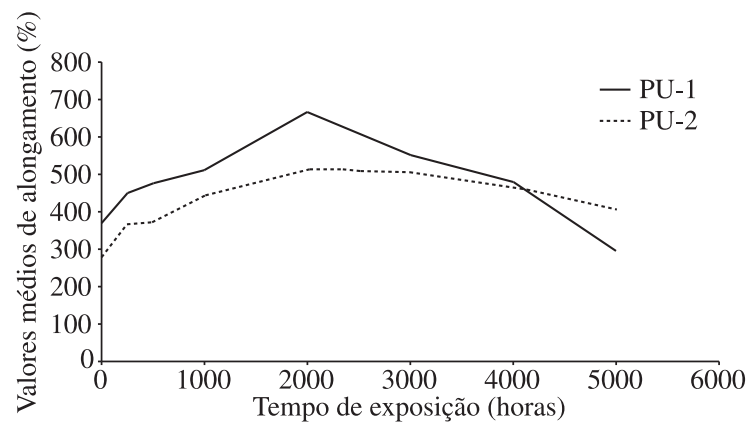

Figura 5. Valores médios de alongamento na ruptura a $70{ }^{\circ} \mathrm{C}$ - Hidrólise.

respectivamente. De um modo em geral, os resultados de alongamento na ruptura para o PU-1 foram maiores que os resultados encontrados para o PU-2 em todos os patamares de temperatura. Esse comportamento era esperado uma vez que a mesma tendência foi observada nos testes de tração realizados antes do teste de envelhecimento. O teor de ligações cruzadas e a presença de segmentos rígidos são fatores que podem explicar o comportamento desses materiais uma vez que a estrutura dos mesmos é de natureza desconhecida como destacado anteriormente.

Para o PU-1, os resultados de alongamento na ruptura apresentaram tendência de crescimento até 4000 horas de exposição, seguida de redução após 5000 horas para os ensaios realizados a 50 e $60{ }^{\circ} \mathrm{C}$. Nos testes realizados a $70{ }^{\circ} \mathrm{C}$, os resultados apresentam elevação até 3000 horas de exposição, seguida de redução significativa após 4000 e 5000 horas de exposição. O PU-2 apresentou tendência de estabilidade nos resultados para todos os patamares de temperatura. Esse 
material mostrou-se mais estável em aplicações marinhas, do ponto de vista da hidrólise.

As Figuras 6 e 7 apresentam separadamente a variação das propriedades de alongamento na ruptura com o tempo de exposição para o PU-1 e PU-2, respectivamente.

As Figuras 8, 9 e 10 apresentam os valores médios de tensão na ruptura para os corpos de prova envelhecidos até 5.000 horas, submetidos a temperatura de 50,60 e $70{ }^{\circ} \mathrm{C}$, respectivamente. De um modo em geral, os resultados de tensão na ruptura para o PU-1 foram inferiores aos resultados encontrados para o PU-2, em todos os patamares de temperatura. Esse comportamento também já era esperado conforme destacado na discussão acima.

Tanto o PU-1, quanto o PU-2 apresentaram decréscimo nos valores médios de tensão na ruptura após 250 horas de exposição em todas as temperaturas do teste. A diminuição dos valores médios de tensão após 250 horas de teste pode estar associada com a absorção de água pelos corpos de prova. Os corpos de

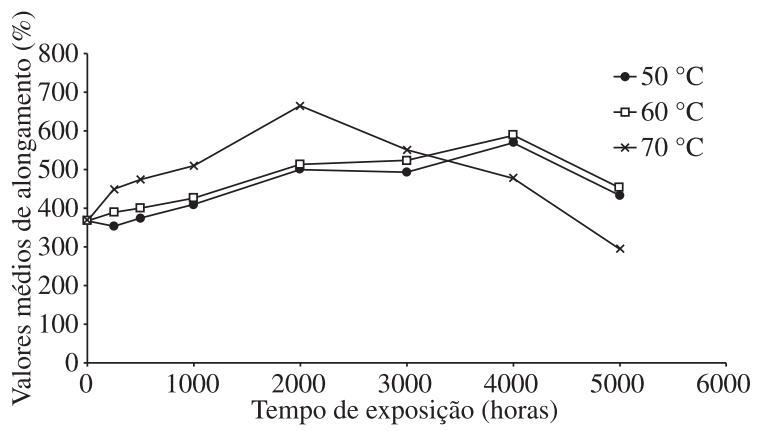

Figura 6. Valores médios de alongamento na ruptura para o PU-1 Hidrólise.

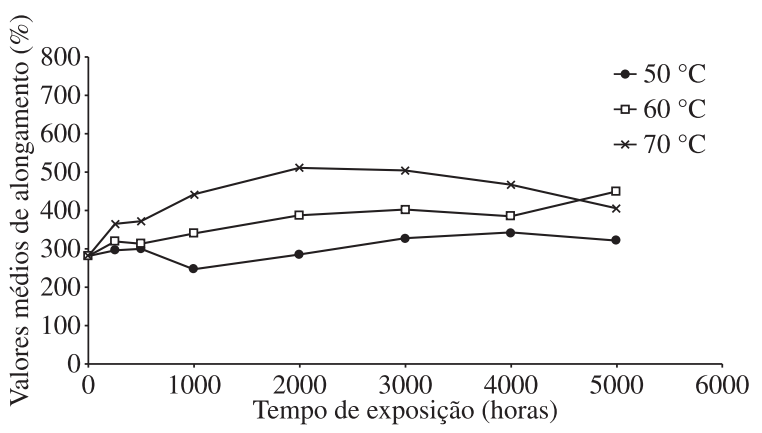

Figura 7. Valores médios de alongamento na ruptura para o PU-2 Hidrólise.

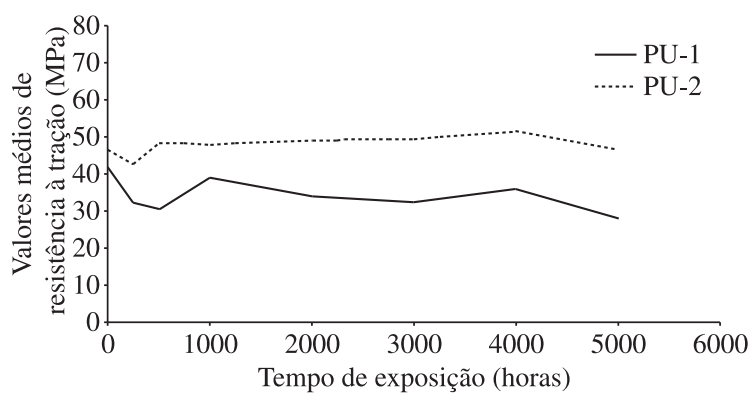

Figura 8. Valores médios de tensão na ruptura a $50{ }^{\circ} \mathrm{C}$ - Hidrólise. prova para o PU-1 e PU-2 tiveram suas massas determinadas antes e após cada período de envelhecimento. Segundo Davies et al. ${ }^{[26]}$ a água pode atuar como plastificante na estrutura do poliuretano, reduzindo assim o módulo de tensão.

Os resultados de envelhecimento por hidrólise obtidos a 50 e $60{ }^{\circ} \mathrm{C}$ apresentaram tendência de estabilidade, de um modo em geral, porém os valores médios de tensão na ruptura realizados a $70{ }^{\circ} \mathrm{C}$ mostraram um acentuado decréscimo, evidenciando assim a ação da hidrólise na redução das propriedades tênseis dos poliuretanos e conseqüente restrição de aplicação dos bend stiffeners neste patamar de temperatura.

\section{Variação de massa para o PU-1}

A Figura 11 ilustra os valores médios de variação de massa encontrados para o PU-1 e para o PU-2. Com base nos resultados, é possível observar que, de um modo em geral, houve um aumento de massa dos corpos de prova para ambos os poliuretanos, sendo um aumento médio de $1,4 \%$ para o PU-1 e 1,2\% para o PU-2. O aumento da massa ocorreu com 250 horas de exposição, mostrando tendência de estabilização até o final do tempo de exposição. Esse aumento de massa está relacionado com a absorção de água pelos corpos de prova.

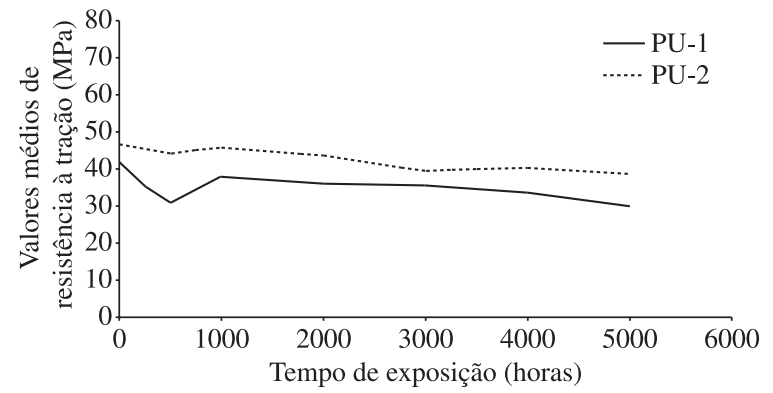

Figura 9. Valores médios de tensão na ruptura a $60{ }^{\circ} \mathrm{C}$ - Hidrólise.

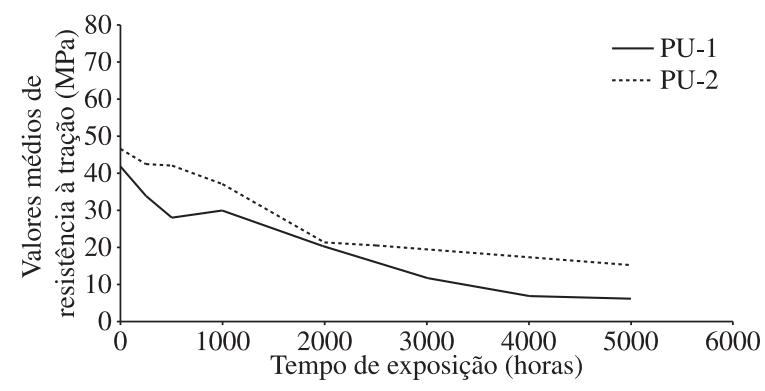

Figura 10. Valores médios de tensão na ruptura a $70{ }^{\circ} \mathrm{C}$ - Hidrólise.

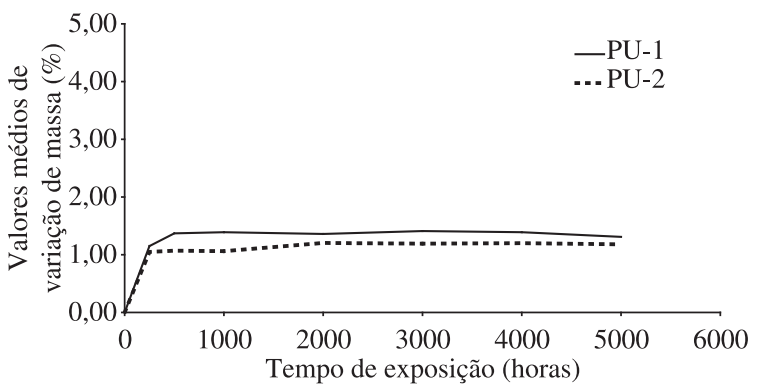

Figura 11. Valores médios de variação de massa para o PU-1 e PU-2. 


\section{Conclusões}

Os valores médios de tensão para o PU-1 e para o PU-2 apresentaram tendência de redução à medida que se aumentou a temperatura de teste para ambos os poliuretanos.

As propriedades mecânicas dos poliuretanos PU-1 e PU-2 podem estar diretamente relacionadas com o teor de ligações cruzadas e segmentos rígidos presentes na estrutura do polímero, uma vez que a estrutura dos materiais são de natureza desconhecida.

Ambos os poliuretanos sofreram degradação significativa no teste de estabilidade hidrolítica a $70{ }^{\circ} \mathrm{C}$.

O PU-1 apresentou aumento de massa de aproximadamente $1,4 \%$ e o PU-2 apresentou aumento de massa de aproximadamente $1,2 \%$ em todos os patamares de temperatura após 250 horas.

A presença da água entre as cadeias do PU-1 influenciou nas propriedades tênseis dos mesmos, uma vez que atuou como plastificante.

\section{Agradecimentos}

A Wellstream International, que possibilitou a realização deste trabalho e ao CNPq pela Bolsa de Produtividade em Pesquisa de F.M.B. Coutinho.

\section{Referências Bibliográficas}

1. Couto, L. O. - "Polímeros Biodegradáveis", Dissertação de Mestrado, Universidade do Estado do Rio de Janeiro, Brasil (2006).

2. Caire, M. - "Análise de Enrijecedores à flexão", Dissertação de Mestrado, Universidade Federal do Rio de Janeiro, Brasil (2005).

3. Hepburn, C. - "Polyurethane Elastomers", Elsevier Science, New York (1992).

4. Saunders, J. H. \& Frisch, K. C. - "Polyurethane chemistry and technology", Interscience Publishers, New York (1987).

5. Vilar, W. D. - "Química e tecnologia de poliuretanos", Grupo Pronor, Rio de Janeiro (1999).

6. Loaec, H.; Monrandeau, F.; Meillat, M. \& Davies, P. Fish. Res., 79, p.210-218 (2006).

7. AMERICAN SOCIETY FOR TESTING AND MATERIALS - ASTM. "ASP 445: materials performance and the deep sea", West Conshohocken (1969).

8. Bowditch, M. R. - Int. J. Adhes. Adhes., 16, p.73-79 (1996).

9. Searle, T. J. and Summerscales, J. - "Review of the durability of marine laminates", in: Reinforced Plastics Durability, cap. 7, Pritchard, G. (ed.)., Woodhead Publishing, Cambridge (1997).
10. Gutierrez, J.; Lelay, F. \& Hoarau, P. - "A study of the Aging of glass fibre-resin composites in a marine environment" in: Proceedings of the third International Conference on Nautical Construction with Composite Materials, p.338-346, Paris, dec (1992).

11. Davies, P.; Mazeas, F. \& Casari, P. - J. Compos. Mater., 35, p.1343-1372, (2001).

12. Kasamori, M.; Funada, Y.; Awaza, K.; Watanabe, Y.; Nakada, M. \& Miyano, Y. - "Accelerated evaluation of mechanical degradation behavior of GFRP in hot water", in: Progress in durability analysis of composite systems, Cardon, A. H.; Fukuda, H. \& Reifsnider, K. (eds.), Taylor \& Francis, London, p.273-277 (1996).

13. Samay, G.; Palotas, L.; Nagy, T. \& Seregely, Z. L. - J. Appl. Polym. Sci., 50, p.359-367 (1992).

14. Burns, J.; Dubbelday, P. S. \& Ting, R. Y. - J. Polym. Sci. Polym. Phys., 28, p.1187-1205 (1990).

15. Murata, S.; Nakajima, T.; Tsuzaki, N.; Yasuda, M. \& Kato, Y. - Polymer Degradation and Stability, 61, p.527-534 (1998).

16. Rutkowska, M.; Krasowska, K.; Heimowska, A.; Steinka, I. \& Janik, H. - Polymer Degradation and Stability, 76, p.233-239 (2002).

17. Campion, R. P.; Thompson, B. \& Harris, J. A. "Elastomers for fluid containment in offshore oil and gas production: guidelines and review", HSE Books, England, p.320 (2005).

18. Celina, M.; Gillen, K. T. \& Assink, R. A. - Polymer Degradation and Stability, 90, p.395-404 (2005).

19. Gillen, K. T.; Berstein, R. \& Derzon, D. K. - Polymer Degradation and Stability, 87, p.567-575 (2005).

20. Gillen, K. T.; Celina, M. \& Berstein, R. - Polymer Degradation and Stability, 82, p.25-35 (2003).

21. Lee, H. S. \& Hsu S. L. - Macromolecules, 22, p.1100-1105 (1989).

22. Lee, H. S.; Wang, Y. K. \& Hsu S. L. - Macromolecules, 20, p.2089-2095 (1987).

23. Lee, H. S.; Wang, Y. K.; Macnight, W. J. \& Hsu, S. L. Macromolecules, 21, p.270-273 (1988).

24. Ashida, K. \& Frisch, K. C. - "International progress in polyurethanes", Technomic Publishing, Lancaster (1993).

25. Rodrigues, J. M. E. - "Preparação de poliuretana à Base de Mamona", Tese de Doutorado, Universidade Federal do Rio Grande do Norte, Brasil (2005).

26. Davies, P. \& Evrard, G. - Polymer Degradation and Stability, 92, p.1455-1464 (2007).

Enviado: $17 / 07 / 09$

Reenviado: 26/10/09

Aceito: 02/12/09

DOI: $10.1590 / \mathrm{S} 0104-14282010005000006$ 